\title{
Electromagnetic Gauge Choice for Scattering of Schrödinger Particle
}

\author{
Andrzej Herdegen@
}

\begin{abstract}
We consider a Schrödinger particle placed in an external electromagnetic field of the form typical for scattering settings in the field theory: $F=F^{\text {ret }}+F^{\text {in }}=F^{\text {adv }}+F^{\text {out }}$, where the current producing $F^{\text {ret/adv }}$ has the past and future asymptotes homogeneous of degree -3 , and the free fields $F^{\text {in/out }}$ are radiation fields produced by currents with similar asymptotic behavior. We show that with appropriate choice of electromagnetic gauge the particle has 'in' and 'out' states reached with no further modification of the asymptotic dynamics. We use a special quantum mechanical evolution 'picture' in which the free evolution operator has well-defined limits for $t \rightarrow \pm \infty$, and thus the scattering wave operators do not need the free evolution counteraction. The existence of wave operators in this setting is established, but the proof of asymptotic completeness is not complete: more precise characterization of the asymptotic behavior of the particle for $|\mathbf{x}|=|t|$ would be needed.
\end{abstract}

Mathematics Subject Classification. Primary 81U99; Secondary 81V10.

\section{Introduction}

Infrared problems are typical for theories with long-range interactions, and extend over wide range of physical settings. They are particularly persistent in the relativistic quantum theory-quantum field theory-where their nature is not only technical, but also conceptual. The standard procedure adopted in mathematically oriented formulations of the quantum electrodynamics (and other theories with long-range interaction) is to use local potentials (of GuptaBleuler type) with adiabatically truncated interaction. One argues that this setting is sufficient to construct (perturbatively) the local algebra of observables of the theory. However, the removal of the cutoff is a singular operation, which has the consequence that the states of the desired theory cannot be 
those in which the truncated theory is constructed. This is well-known and generally accepted. But one can also ask, whether the truncation of interaction does not remove some structure from the theory in an irreversible way; the algebraic equivalence of local algebras, for all cutoff functions equal to one in the considered region, is based on an interpolating relation, which becomes singular in the limit of the function tending to unity on the whole spacetime. ${ }^{1}$

Considerations similar to those described above have motivated the present author, many years ago, to attempt to include, from the beginning, the long-range degrees of freedom of the theory in the description. These attempts went in two main directions: (i) construction of a nonlocal electromagnetic potential in which scattering of a Dirac particle is infrared nonsingular (see [4] for the analysis at the level of classical fields); and (ii) extension of the algebra of the free quantum electromagnetic field which includes long-range degrees of freedom and which is thought of as an asymptotic algebra, potentially starting point for perturbation calculus (see [5] and a recent synopsis [6]).

The present article is a further test of the idea mentioned in (i) above. In article [4] I have considered the scattering of the classical Dirac field in an external electromagnetic field of the type supposed to be present in full interacting theory. It was shown that if an appropriate (nonlocal, in general) electromagnetic gauge is chosen, then the Dirac field has a well-defined asymptote in remote future (and past) inside the lightcone. This asymptote is reached without further corrections of asymptotic dynamics, as usually employed in longrange scattering. Moreover, the scattered outgoing Dirac field is constructed with the use of this asymptote. The hope behind this analysis is that a similar construction in quantum case could similarly relieve some of the infrared problems.

The article mentioned above lacks the discussion of the asymptotic behavior of the Dirac field on the whole hyperplanes of constant time, for this time tending to infinity. In the present paper we analyze this question in the case of nonrelativistic Schrödinger particle. We show the existence of asymptotic velocity operators and of isometric wave operators. The null asymptotic behavior of radiation fields (and their potentials), together with all their derivatives, is only of (1/time) type, which breaks the usual assumptions imposed on time-decaying potentials considered in Schrödinger scattering (see $[2,11]$ ). In the present setting this behavior has prevented the proof of asymptotic completeness. Whether this can be overcome is an open question. ${ }^{2}$

The choice of gauge found appropriate for the problem of [4] was such that $x \cdot A(x)$ (Minkowski product of the position vector with the potential) vanishes sufficiently fast in remote future (and past) inside the lightcone $\left(x^{2}>0\right)$. Here we shall construct an appropriate gauge in the whole spacetime and will see that its behavior inside the lightcone is of the same type as before.

\footnotetext{
${ }^{1}$ For recent results on adiabatic limit in quantum field theory, as well as a review of literature, see [1].

${ }^{2}$ Long-range scattering in the position representation has been discussed before, see, e.g., $[3,11]$, but not for the context considered here, and with no relation to the choice of electromagnetic gauge.
} 
The method used for our analysis is the transformation of the time evolution from the Schrödinger picture to a new 'picture', which we describe in Sect. 2, and in which the state vector of a particle tends to its spacetime asymptotic forms in asymptotic times. As it turns out, the simplest choice of such transformation is the well-known Niederer transformation to the harmonic oscillator system [8]. In Sect. 3 this oscillator is placed in electromagnetic field, and in Sect. 4 transformed back to the Schrödinger picture. In order to construct the time-dependent Hamiltonian, and the evolution it generates, we follow an article by Yajima [12] in its use of the Kato theorem. ${ }^{3}$ In Sect. 5 we discuss in detail the relation between potentials and fields in the two pictures and define a gauge appropriate for the description of scattering along the lines described above. Section 6 contains theorems on scattering in oscillator picture. Reformulation of these results in natural spacetime terms and some final remarks are given in Sect. 7. Appendix A discusses some relations between domains of operators needed in the main text. In Appendix B, we discuss the scope of electromagnetic fields admitted in the article, and analyze, for completeness, their spacetime estimates. Appendix C clarifies a particular differentiation employed in Sect. 6.

\section{Harmonic Oscillator Picture}

Let $\mathcal{H}=L^{2}\left(\mathbb{R}^{3}\right)$ and denote $H_{0}=-\frac{1}{2} \Delta$, the free particle Hamiltonian with the corresponding evolution operator $U_{0}(t, s) .{ }^{4}$ The free particle wave packet $\psi(t)=U_{0}(t, 0) \psi$ is given by the Fourier representation

$$
\psi(t, \mathbf{x})=(2 \pi)^{-3 / 2} \int \exp \left[-\frac{i}{2} t|\mathbf{p}|^{2}+i \mathbf{p} \cdot \mathbf{x}\right] \hat{\psi}(\mathbf{p}) \mathrm{d}^{3} p,
$$

and for $\hat{\psi}$ regular enough, by the use of stationary phase method, has the following asymptotic forms for $|t| \rightarrow \infty$ :

$$
\psi(t,|t| \mathbf{y}) \sim|t|^{-3 / 2} \mathrm{e}^{\mp i 3 \pi / 4} \exp \left[\frac{i}{2} t|\mathbf{y}|^{2}\right] \hat{\psi}( \pm \mathbf{y}) \quad \text { for } \quad t \rightarrow \pm \infty .
$$

We would like to find a new evolution 'picture', in which the state vector of the particle has well-defined limits for $t \rightarrow \pm \infty$. Using the asymptotic forms (1) as a guideline, we define for $t \in \mathbb{R}$ the following transformation in $\mathcal{H}$ :

$$
[N(t) \chi](\mathbf{x})=\langle t\rangle^{-3 / 2} \exp \left[\frac{i t}{2}\left(\frac{|\mathbf{x}|}{\langle t\rangle}\right)^{2}\right] \chi\left(\frac{\mathbf{x}}{\langle t\rangle}\right),
$$

\footnotetext{
${ }^{3}$ There is also some similarity in the use of two time evolutions related by unitary transformations. However, the transformations are quite different: It is a pure gauge transformation in [12], while here it is the transformation to the new 'picture' mentioned above. Also, the aims of these operations are quite different: Yajima's goal is to include electromagnetic fields as singular as possible (both locally, and in infinity), while here we are interested in scattering theory.

${ }^{4}$ We set $\hbar=1, c=1$ and use dimensionless rescaled quantities; to recover physical quantities, one should substitute $(t, \mathbf{x}) \mapsto(m t, m \mathbf{x})$, with $m$ the mass of the particle. Also, the electromagnetic potentials to appear later should be multiplied by $q / m$, with $q$ the charge of the particle.
} 
where $\langle t\rangle>0$ is a $C^{1}$-function of $t$, such that $\langle t\rangle /|t| \rightarrow 1$ for $|t| \rightarrow \infty$, whose exact form is to be determined. This is a unitary transformation and the conjugate transformation is

$$
\left[N(t)^{*} \psi\right](\mathbf{y})=\langle t\rangle^{3 / 2} \exp \left[-\frac{i}{2} t|\mathbf{y}|^{2}\right] \psi(\langle t\rangle \mathbf{y})
$$

We use the family of operators $N(t)$ to transform the Schrödinger state vectors and observables, respectively, by

$$
\psi_{S}(t) \mapsto \psi_{N}(t)=N(t)^{*} \psi_{S}(t), \quad A_{S}(t) \mapsto A_{N}(t)=N(t)^{*} A_{S}(t) N(t) .
$$

The evolution operator for $\psi_{N}(t)$ :

$$
U_{0 N}(t, s)=N(t)^{*} U_{0}(t, s) N(s)
$$

is strongly continuous, and for $\chi \in C_{0}^{\infty}\left(\mathbb{R}^{3}\right)$ the vector $U_{0 N}(t, s) \chi$ is strongly differentiable in $t$ and in $s$, and a straightforward calculation shows that

$$
i \partial_{t} U_{0 N}(t, s) \chi=H_{0 N}(t) U_{0 N}(t, s) \chi,
$$

where (with $\left.\langle t\rangle^{\prime}=\mathrm{d}\langle t\rangle / \mathrm{d} t\right)$

$$
\begin{aligned}
H_{0 N}(t) & =N(t)^{*} H_{0} N(t)-i N(t)^{*} \partial_{t} N(t) \\
& =\frac{1}{2\langle t\rangle^{2}}\left(\left[\mathbf{p}^{2}+\left(\langle t\rangle^{2}-t^{2}\right) \mathbf{x}^{2}\right]+\left(t-\langle t\rangle\langle t\rangle^{\prime}\right)\left[\mathbf{x} \cdot \mathbf{p}+\mathbf{p} \cdot \mathbf{x}+2 t \mathbf{x}^{2}\right]\right)
\end{aligned}
$$

here and in what follows $\mathbf{p}=-i \boldsymbol{\partial}$. A large class of functions $\langle t\rangle$ for which $t-\langle t\rangle\langle t\rangle^{\prime}$ tends to zero and $\langle t\rangle^{2}-t^{2}$ tends to a constant sufficiently fast would fulfil our demands formulated after (1). However, the simplest choice is to demand $t-\langle t\rangle\langle t\rangle^{\prime}=0$, in which case $\langle t\rangle^{2}=t^{2}+\kappa^{2}$, where $\kappa^{2}$ is a constant. The choice of this constant is physically irrelevant, and as our variables are dimensionless, the simplest choice is $\kappa=1$, so that $N(0)=\mathbb{1}$. The new picture Hamiltonian is now

$$
H_{0 N}(t)=\frac{1}{2\langle t\rangle^{2}}\left[\mathbf{p}^{2}+\mathbf{x}^{2}\right], \quad\langle t\rangle=\sqrt{t^{2}+1},
$$

and the choice of $\langle t\rangle$ holds in the rest of the article. If we now define

$$
u_{0}(\tau, \sigma)=U_{0 N}(\tan \tau, \tan \sigma),
$$

then

$$
u_{0}(\tau, \sigma)=\exp \left[-i(\tau-\sigma) h_{0}\right], \quad h_{0}=\frac{1}{2}\left[\mathbf{p}^{2}+\mathbf{x}^{2}\right]
$$

The evolution of the free particle state over $t \in(-\infty,+\infty)$ corresponds now to the evolution over $\tau \in(-\pi / 2,+\pi / 2)$, thus over half the period of the harmonic oscillator. We shall call this new description of evolution the harmonic oscillator picture. The scattering change of state is described in this picture by the operator

$$
\mathrm{s}_{\tau \nearrow / \pi / 2} u_{0}(+\tau,-\tau)=u_{0}(+\pi / 2,-\pi / 2)=\exp \left[-i \pi h_{0}\right]=i P,
$$

where $P$ is the parity operator $[P \chi](\mathbf{x})=\chi(-\mathbf{x})$. This formula is in agreement with the asymptotic forms (1).

The transformation $N(t)$ leading from a free particle to an harmonic oscillator has been discovered much earlier by Niederer [8]. It is interesting to note 
that his original derivation was a result of group-theoretical considerations, with no relation to scattering theory. Although the Niederer transformation has found numerous applications in literature, to our knowledge it has not appeared in the scattering of a Schrödinger particle context before. Which, if true, would be quite surprising, if one notes its striking similarity to the asymptotic form (1).

We shall later see that the above relation extends to a system placed in external electromagnetic field: a charged particle dynamics becomes a charged oscillator in the new picture (with electromagnetic potentials appropriately transformed). For technical reasons, we find it convenient to start our discussion in the oscillator picture, and only then transform into Schrödinger picture.

\section{Harmonic Oscillator in Electromagnetic Field}

We consider in $\mathcal{H}=L^{2}\left(\mathbb{R}^{3}\right)$ the Hamiltonian

$$
h=\frac{1}{2}\left(\boldsymbol{\pi}^{2}+\mathbf{x}^{2}\right)+v, \quad \boldsymbol{\pi}=\mathbf{p}-\mathbf{a},
$$

where $\mathbf{a}(\tau, \mathbf{x})$ and $v(\tau, \mathbf{x})$ are electromagnetic potentials. This Hamiltonian may be given the precise meaning for a wide class of potentials (see [12]), but for our purposes it is sufficient to consider the following setting.

First note, that for vanishing potentials the corresponding 'unperturbed' harmonic oscillator Hamiltonian $h_{0}=\frac{1}{2}\left(\mathbf{p}^{2}+\mathbf{x}^{2}\right)$ is determined by the closed quadratic form ${ }^{5}$

$$
\begin{aligned}
q_{0}(\varphi, \psi) & =\frac{1}{2}(\mathbf{p} \varphi, \mathbf{p} \psi)+\frac{1}{2}(\mathbf{x} \varphi, \mathbf{x} \psi), \\
\mathcal{D}\left(q_{0}\right) & =\{\psi \in \mathcal{H} \mid \mathbf{p} \psi \in \mathcal{H}, \mathbf{x} \psi \in \mathcal{H}\}=\mathcal{D}(\mathbf{p}) \cap \mathcal{D}(\mathbf{x})=\mathcal{D}\left(h_{0}^{1 / 2}\right)
\end{aligned}
$$

and the following relations hold

$$
\mathcal{D}\left(h_{0}\right)=\mathcal{D}\left(\mathbf{p}^{2}\right) \cap \mathcal{D}\left(\mathbf{x}^{2}\right) \subseteq \mathcal{D}(\mathbf{x} \cdot \mathbf{p}+\mathbf{p} \cdot \mathbf{x})
$$

(see Appendix A). ${ }^{6}$ Moreover, $C_{0}^{\infty}\left(\mathbb{R}^{3}\right)$ is a form-core and a core for $h_{0}$ (see Thm. X.28 in [10]).

We also note an elementary inequality which will be used below. Let $\psi(\tau) \in \mathcal{H}$ depend differentiably on a real parameter $\tau$. Then

$$
\left|\partial_{\tau}\|\psi(\tau)\|\right| \leq\left\|\partial_{\tau} \psi(\tau)\right\|
$$

To show this, note that $\partial_{\tau}\|\psi(\tau)\|^{2}=2 \operatorname{Re}\left(\psi(\tau), \partial_{\tau} \psi(\tau)\right)$ and use the Schwarz inequality for the RHS.

Proposition 1. Let a and $v$ be in $L^{\infty}\left(\mathbb{R}^{3}\right)$ (for each fixed time) and define the quadratic form

$$
q(\varphi, \psi)=\frac{1}{2}(\boldsymbol{\pi} \varphi, \boldsymbol{\pi} \psi)+\frac{1}{2}(\mathbf{x} \varphi, \mathbf{x} \psi)+(\varphi, v \psi) \equiv q_{0}(\varphi, \psi)+\rho(\varphi, \psi),
$$

\footnotetext{
${ }^{5}$ Conditions like $\mathbf{p} \psi \in \mathcal{H}$ and $\mathbf{x} \psi \in \mathcal{H}$ below, and similar other to appear further with vector quantities on the LHS, are meant to hold component-wise.

${ }^{6}$ It is quite surprising that the characterization of the harmonic oscillator domain given by the equality in (2) is rather hard to find in standard textbook discussions of the harmonic oscillator.
} 
with the form-domain $\mathcal{D}(q)=\mathcal{D}\left(q_{0}\right)$. Then $q$ is a closed form corresponding to the unique self-adjoint operator $h$, for which $C_{0}^{\infty}\left(\mathbb{R}^{3}\right)$ is a form-core. If $\alpha>$ $\|v\|_{\infty}$, then $h+\alpha \mathbb{1}$ is positive, and $\mathcal{D}\left((h+\alpha \mathbb{1})^{1 / 2}\right)=\mathcal{D}\left(q_{0}\right)$.

If, in addition, $(\boldsymbol{\partial} \cdot \mathbf{a})$ is also in $L^{\infty}\left(\mathbb{R}^{3}\right)$, then $\mathcal{D}(h)=\mathcal{D}\left(h_{0}\right)$, and $C_{0}^{\infty}\left(\mathbb{R}^{3}\right)$ is a domain of essential self-adjointness of $h$.

Proof. For $\varphi \in \mathcal{D}\left(q_{0}\right)$ one has

$$
|\rho(\varphi, \varphi)| \leq\|\mathbf{a} \varphi\|\|\mathbf{p} \varphi\|+\left(\varphi,\left[\frac{1}{2} \mathbf{a}^{2}+|v|\right] \varphi\right) \leq \frac{1}{2} q_{0}(\varphi, \varphi)+c_{1}\|\varphi\|^{2} .
$$

Therefore, the first part of the theorem follows by the KLMN theorem (see, e.g., [10], Thm. X.17). If, in addition, $(\boldsymbol{\partial} \cdot \mathbf{a}) \in L^{\infty}\left(\mathbb{R}^{3}\right)$, then for $\varphi \in \mathcal{D}\left(q_{0}\right)$ and $\psi \in \mathcal{D}\left(h_{0}\right)$ we have $\rho(\varphi, \psi)=(\varphi, r \psi)$ with

$$
r=h-h_{0}=-\mathbf{a} \cdot \mathbf{p}+\frac{i}{2}(\boldsymbol{\partial} \cdot \mathbf{a})+\frac{1}{2} \mathbf{a}^{2}+v,
$$

So

$$
\|r \psi\| \leq\left(\|\mathbf{a}\|_{\infty}\|\psi\|^{1 / 2}\right)\left(\|\mathbf{p} \psi\|\|\psi\|^{-1 / 2}\right)+c_{2}\|\psi\| \leq \frac{1}{2}\left\|h_{0} \psi\right\|+c_{3}\|\psi\| .
$$

Thus the second part of the thesis follows by the Kato-Rellich theorem.

The existence of the corresponding evolution operators is assured under the conditions of the following theorem. Here and in the rest of the article, the overdot denotes differentiation with respect to time in the oscillator picture.

Theorem 2. For an interval $I \subset \mathbb{R}$, let the functions $\mathbf{a}, \boldsymbol{\partial} \cdot \mathbf{a}, \dot{\mathbf{a}}, \boldsymbol{\partial} \cdot \dot{\mathbf{a}}, v$ and $\dot{v}$ be in $L^{\infty}\left(I \times \mathbb{R}^{3}\right)$. Then for $\tau, \sigma \in I$ :

(i) all $h(\tau)$ satisfy Proposition 1;

(ii) there exists the unique unitary propagator $u(\tau, \sigma)$ for the family $h(\tau)$, strongly continuous in $(\tau, \sigma)$, with the following properties:

(a) $u(\tau, \sigma) \mathcal{D}\left(h_{0}\right)=\mathcal{D}\left(h_{0}\right)$;

(b) for $\psi \in \mathcal{D}\left(h_{0}\right)$ the map $(\tau, \sigma) \mapsto u(\tau, \sigma) \psi$ is of class $C^{1}$ in the strong sense and

$$
\begin{aligned}
& i \partial_{\tau} u(\tau, \sigma) \psi=h(\tau) u(\tau, \sigma) \psi, \\
& i \partial_{\sigma} u(\tau, \sigma) \psi=-u(\tau, \sigma) h(\sigma) \psi .
\end{aligned}
$$

Proof. For $\psi \in \mathcal{D}\left(h_{0}\right)$ we have

$$
\dot{h} \psi=\dot{r} \psi=\left[-\dot{\mathbf{a}} \cdot \mathbf{p}+\frac{i}{2}(\boldsymbol{\partial} \cdot \dot{\mathbf{a}})+\dot{\mathbf{a}} \cdot \mathbf{a}+\dot{v}\right] \psi,
$$

see (4), so estimating as in (5) we obtain $\|\dot{h} \psi\| \leq \frac{1}{2}\left\|h_{0} \psi\right\|+c_{4}\|\psi\|$ and

$$
\|[h(\tau)-h(\sigma)] \psi\| \leq|\tau-\sigma|\left(\frac{1}{2}\left\|h_{0} \psi\right\|+c_{4}\|\psi\|\right) .
$$

Next, using (5) we find

$$
\left\|h_{0} \psi\right\|=2\|[h-r] \psi\|-\left\|h_{0} \psi\right\| \leq 2\left(\|h \psi\|+c_{3}\|\psi\|\right),
$$

SO

$$
\|\dot{h} \psi\| \leq\|h \psi\|+c_{5}\|\psi\|
$$

Let us denote

$$
\|\psi\|_{\tau} \equiv\left[\|h(\tau) \psi\|^{2}+\|\psi\|^{2}\right]^{1 / 2}
$$


-the graph norm of $h(\tau)$. The use of (3) now gives

$$
\left|\partial_{\tau}\|\psi\|_{\tau}^{2}\right| \leq 2\|h \psi\|\|\dot{h} \psi\| \leq 2 c\|\psi\|_{\tau}^{2}
$$

for some $c>0$. This implies $\|\psi\|_{\tau} \leq e^{c|\tau-\sigma|}\|\psi\|_{\sigma}$, and the way is paved for the application of the Kato theorem in the form given by Theorem 3.2 in [12]. Denote $\mathcal{Y}=\mathcal{D}\left(h_{0}\right)$ equipped with the graph norm of $h_{0}$, and $\mathcal{X}=\mathcal{H}$. Then (8) says that $h(\tau): \mathcal{Y} \mapsto \mathcal{X}$ is norm-continuous. Moreover, let $\mathcal{Y}_{\tau}$ be $\mathcal{D}\left(h_{0}\right)$ equipped with the graph norm $\|\cdot\|_{\tau}$, and identify $A(\tau)=h(\tau)$. Then all conditions of this theorem are satisfied and one obtains $u(\tau, \sigma)$ with the stated properties.

\section{From Oscillator to Schrödinger Picture}

The way back to the Schrödinger picture is achieved as follows.

Theorem 3. Let a and $v$ satisfy the assumptions of Theorem 2 on each compact interval $I \subset(-\pi / 2,+\pi / 2)$. Denote

$$
\begin{aligned}
& \mathbf{A}(t, \mathbf{x})=\langle t\rangle^{-1} \mathbf{a}\left(\arctan t,\langle t\rangle^{-1} \mathbf{x}\right), \\
& V(t, \mathbf{x})=\langle t\rangle^{-2}\left[v\left(\arctan t,\langle t\rangle^{-1} \mathbf{x}\right)+\langle t\rangle^{-1} t \mathbf{x} \cdot \mathbf{a}\left(\arctan t,\langle t\rangle^{-1} \mathbf{x}\right)\right], \\
& U(t, s)=N(t) u(\arctan t, \arctan s) N(s)^{*} .
\end{aligned}
$$

Then $\mathbb{R}^{2} \ni(t, s) \mapsto U(t, s)$ is a strongly continuous family of unitary evolution operators, with the properties:

(i) $U(t, s) \mathcal{D}\left(h_{0}\right)=\mathcal{D}\left(h_{0}\right)$;

(ii) for $\psi \in \mathcal{D}\left(h_{0}\right)$ the map $(t, s) \mapsto U(t, s) \psi$ is of class $C^{1}$ in the strong sense and

$$
i \partial_{t} U(t, s) \psi=H(t) U(t, s) \psi, \quad i \partial_{s} U(t, s) \psi=-U(t, s) H(s) \psi,
$$

where

$$
H(t)=\frac{1}{2} \boldsymbol{\Pi}(t)^{2}+V(t), \quad \boldsymbol{\Pi}=\mathbf{p}-\mathbf{A} .
$$

Operators $H(t)$ are essentially self-adjoint on $C_{0}^{\infty}\left(\mathbb{R}^{3}\right)$.

The inverse relations of potentials are

$$
\begin{aligned}
& \mathbf{a}(\tau, \mathbf{x})=\left.\langle t\rangle \mathbf{A}(t,\langle t\rangle \mathbf{x})\right|_{t=\tan \tau} \\
& v(\tau, \mathbf{x})=\left.\langle t\rangle^{2}[V(t,\langle t\rangle \mathbf{x})-(t /\langle t\rangle) \mathbf{x} \cdot \mathbf{A}(t,\langle t\rangle \mathbf{x})]\right|_{t=\tan \tau} .
\end{aligned}
$$

Proof. Taking into account relations (2) one finds $N(s)^{*} \mathcal{D}\left(h_{0}\right)=\mathcal{D}\left(h_{0}\right)$ and $N(t) \mathcal{D}\left(h_{0}\right)=\mathcal{D}\left(h_{0}\right)$, which proves (i). For $\psi \in \mathcal{D}\left(h_{0}\right)$ and $F$-the operator of multiplication by the function $F(\mathbf{x})$, we have

$$
\begin{aligned}
& {\left[N(t) F N(t)^{*} \psi\right](\mathbf{x})=F(\mathbf{x} /\langle t\rangle) \psi(\mathbf{x}),} \\
& {\left[N(t) \mathbf{p} N(t)^{*} \psi\right](\mathbf{x})=[\langle t\rangle \mathbf{p}-(t /\langle t\rangle) \mathbf{x}] \psi(\mathbf{x}),}
\end{aligned}
$$

so a straightforward calculation gives

$$
\begin{aligned}
& {\left[N(t) h(\arctan t) N(t)^{*} \psi\right](\mathbf{x})} \\
& \quad=\langle t\rangle^{2}\left[\frac{1}{2}(\mathbf{p}-A(t, \mathbf{x}))^{2}+V(t, \mathbf{x})\right] \psi(\mathbf{x})+\frac{1}{2}\left[\mathbf{x}^{2}-t(\mathbf{x} \cdot \mathbf{p}+\mathbf{p} \cdot \mathbf{x})\right] \psi(\mathbf{x}),
\end{aligned}
$$


with $\mathbf{A}$ and $V$ given in the thesis. On the other hand

$$
-i\left[N(t) \partial_{t} N(t)^{*} \psi\right](\mathbf{x})=\frac{1}{2}\langle t\rangle^{-2}\left[-\mathbf{x}^{2}+t(\mathbf{x} \cdot \mathbf{p}+\mathbf{p} \cdot \mathbf{x})\right] \psi(\mathbf{x}) .
$$

Thus equations (11) are satisfied with

$$
H(t)=\langle t\rangle^{-2} N(t) h(\arctan t) N(t)-i N(t) \partial_{t} N(t)=\frac{1}{2} \boldsymbol{\Pi}(t)^{2}+V(t) .
$$

For each $t$ the norm $\|\mathbf{A}(t, .)\|_{\infty}$ is finite, and if $\|V(t, .)\|_{\infty}$ is finite as well, then the essential self-adjointness follows easily in standard way (as in the proof of Proposition 1). In general, there is only $\left\|\langle|\mathbf{x}|\rangle^{-1} V(t, .)\right\|_{\infty}<\infty$ (due to the $\mathbf{x} \cdot \mathbf{a}$ term in $V$ ), but then the use of the Leinfelder-Simader theorem (Thm. 4 in [7]) leads to the same conclusion.

\section{Electromagnetic Fields and Gauges}

We shall now discuss in detail the relation between $(V, \mathbf{A})$ and $(v, \mathbf{a})$ defined in (9). We assume that all differentiations to appear may be performed, but in the following theorem the assumptions of Theorem 3 are not needed.

Proposition 4. Let $(V, \mathbf{A})$ and $(v, \mathbf{a})$ be related by $(9)$. Denote the electric and magnetic fields of these potentials by $(\mathbf{E}, \mathbf{B})$ and $(\mathbf{e}, \mathbf{b})$, respectively. Then the following is satisfied:

(i) The gauge transformation

$$
\mathbf{A}_{\Lambda}(t, \mathbf{x})=\mathbf{A}(t, \mathbf{x})-\boldsymbol{\partial} \Lambda(t, \mathbf{x}), \quad V_{\Lambda}(t, \mathbf{x})=V(t, \mathbf{x})+\partial_{t} \Lambda(t, \mathbf{x}),
$$

is equivalent to the transformation

$$
\mathbf{a}_{\lambda}(\tau, \mathbf{x})=\mathbf{a}(\tau, \mathbf{x})-\boldsymbol{\partial} \lambda(\tau, \mathbf{x}), \quad v_{\lambda}(\tau, \mathbf{x})=v(\tau, \mathbf{x})+\partial_{\tau} \lambda(\tau, \mathbf{x})
$$

where $\lambda(\tau, \mathbf{x})=\left.\Lambda(t,\langle t\rangle \mathbf{x})\right|_{t=\tan \tau}$.

(ii) The electromagnetic fields are related by:

$$
\begin{aligned}
\mathbf{e}(\tau, \mathbf{x}) & =\left[\langle t\rangle^{3} \mathbf{E}(t,\langle t\rangle \mathbf{x})+t\langle t\rangle^{2} \mathbf{x} \times \mathbf{B}(t,\langle t\rangle \mathbf{x})\right]_{t=\tan \tau}, \\
\mathbf{b}(\tau, \mathbf{x}) & =\left.\langle t\rangle^{2} \mathbf{B}(t,\langle t\rangle \mathbf{x})\right|_{t=\tan \tau} .
\end{aligned}
$$

(iii) For given $(v, \mathbf{a})$ choose the gauge function as

$$
\lambda_{\mathrm{e}}(\tau, \mathbf{x})=-\int_{0}^{\tau} v(\rho, \mathbf{x}) \mathrm{d} \rho+\lambda_{\mathrm{e}}(0, \mathbf{x}),
$$

where $\lambda_{\mathrm{e}}(0, \mathbf{x})$ constitutes the remaining freedom in the definition. Then:

$$
v_{\mathrm{e}}(\tau, \mathbf{x})=0, \quad \mathbf{a}_{\mathrm{e}}(\tau, \mathbf{x})=\mathbf{a}_{\mathrm{e}}(0, \mathbf{x})-\int_{0}^{\tau} \mathbf{e}(\rho, \mathbf{x}) \mathrm{d} \rho,
$$

which we shall call an a-gauge. In these gauges the assumptions of Theorem 3 are reduced to the following: $\mathbf{a}_{\mathrm{e}}(0), \boldsymbol{\partial} \cdot \mathbf{a}_{\mathrm{e}}(0) \in L^{\infty}\left(\mathbb{R}^{3}\right)$ and $\mathbf{e}, \boldsymbol{\partial} \cdot \mathbf{e} \in L^{\infty}\left(I \times \mathbb{R}^{3}\right)$ for each compact interval $I \subset(-\pi / 2,+\pi / 2)$.

(iv) In all a-gauges the four-potential $A^{a}(x)=(V(t, \mathbf{x}), \mathbf{A}(t, \mathbf{x}))$ satisfies

$$
\hat{x} \cdot A(x)=0, \quad \hat{x}=x+\left(\left(x^{0}\right)^{-1}, \mathbf{0}\right)
$$

(there is no singularity at $x^{0}=0$ ). 
Proof. All properties follow by simple calculations, which we leave to the reader.

Preparing to discuss scattering, we need to formulate the asymptotic behavior of potentials and fields: for upper case fields in the limit $|t| \rightarrow \infty$, and the corresponding behavior of lower case fields in the limit $|\tau| \rightarrow \pi / 2$. It will be convenient to denote

$$
\mathbf{C}(t, \mathbf{x})=t \mathbf{E}(t, \mathbf{x})+\mathbf{x} \times \mathbf{B}(t, \mathbf{x}),
$$

so then

$$
\mathbf{e}(\tau, \mathbf{x})=[t\langle t\rangle \mathbf{C}(t,\langle t\rangle \mathbf{x})+\langle t\rangle \mathbf{E}(t,\langle t\rangle \mathbf{x})]_{t=\tan \tau} .
$$

In what follows, we shall always use a-gauges, so we only need to consider the fields $\mathbf{E}, \mathbf{C}$ and $\mathbf{e}$. In Appendix B we summarize the asymptotic properties of electromagnetic fields in scattering settings in Minkowski space. For $\mathbf{E}$ and C, it will be sufficient to note

$$
|\mathbf{E}(t, \mathbf{x})| \leq \text { const }, \quad|\mathbf{C}(t, \mathbf{x})| \leq \frac{\text { const }}{1+|t|+|\mathbf{x}|},
$$

while for divergence of $\mathbf{C}$ we shall need the bound

$$
|\partial \cdot \mathbf{C}(t, \mathbf{x})| \leq \frac{\text { const }}{(1+|t|+|\mathbf{x}|)(1+|| t|-| \mathbf{x}||)^{\gamma}}
$$

for some $1 \geq \gamma>0$. A straightforward calculation with the use of (16) shows that these relations imply the following lower case fields bounds:

$$
|\mathbf{e}(\tau, \mathbf{x})| \leq \frac{\text { const }}{\cos \tau}, \quad|\boldsymbol{\partial} \cdot \mathbf{e}(\tau, \mathbf{x})| \leq \frac{\text { const }}{(\cos \tau)^{2-\gamma}(\cos \tau+\| \sin \tau|-| \mathbf{x}||)^{\gamma}},
$$

where in the last formula we have taken into account that $\boldsymbol{\partial} \cdot \mathbf{E}(t, \mathbf{x})=4 \pi \rho(t, \mathbf{x})$ (the charge density), which gives a contribution to $\boldsymbol{\partial} \cdot \mathbf{e}$ bounded by a constant, which is less restricting than the term in (19).

While reading the next section, the reader is asked to note that the bounds (19) are sufficient to satisfy the assumptions of Theorems 5 and 6 , but for Theorem 7 the second bound in (19) would have to be replaced by

$$
|\boldsymbol{\partial} \cdot \mathbf{e}(\tau, \mathbf{x})| \leq \frac{\text { const }}{(\cos \tau)^{2-\gamma}} .
$$

\section{Scattering in Oscillator picture}

Turning to the discussion of scattering, we start again with the oscillator picture. With increasingly restrictive assumptions, we shall prove:

(i) existence of asymptotic position,

(ii) existence of wave operators, and

(iii) their unitarity (asymptotic completeness). 
From now on, we shall use only a-gauges defined by (13), and we omit the subscript e.

For any operator $k=k(\tau)$ with $\mathcal{D}\left(h_{0}\right) \subseteq \mathcal{D}(k(\tau))$ we denote

$$
\tilde{k}=\tilde{k}(\tau)=u(0, \tau) k(\tau) u(\tau, 0), \quad \text { so } \quad \mathcal{D}\left(h_{0}\right) \subseteq \mathcal{D}(\tilde{k}(\tau)) .
$$

We assume that the assumptions of Theorem 3, as reformulated in Proposition 4 (iii), are satisfied. Then for $\psi \in \mathcal{D}\left(h_{0}\right)$, following the remarks in Appen$\operatorname{dix} \mathrm{C}$, one finds

$$
\begin{aligned}
\dot{\tilde{h}} \psi & =\frac{1}{2}[\tilde{\mathbf{e}} \cdot \tilde{\pi}+\tilde{\pi} \cdot \tilde{\mathbf{e}}] \psi, \\
\dot{\tilde{\mathbf{x}}} \psi & =\tilde{\pi} \psi, \\
\partial_{\tau} f(\tilde{\mathbf{x}}) \psi & =(\partial f)(\tilde{\mathbf{x}}) \cdot \tilde{\pi} \psi-\frac{i}{2}(\Delta f)(\tilde{\mathbf{x}}) \psi,
\end{aligned}
$$

where $f$ is any smooth bounded function with bounded derivatives. Using (22) we obtain

$$
\left|\partial_{\tau}\left\|\tilde{h}^{1 / 2} \psi\right\|^{2}\right|=|(\psi, \dot{\tilde{h}} \psi)| \leq\|\tilde{\mathbf{e}} \psi\|\|\tilde{\boldsymbol{\pi}} \psi\| \leq \sqrt{2}\|\tilde{\mathbf{e}} \psi\|\left\|\tilde{h}^{1 / 2} \psi\right\|,
$$

so

$$
\left\|\tilde{h}(\tau)^{1 / 2} \psi\right\|-\left\|h(0)^{1 / 2} \psi\right\| \mid \leq \frac{1}{\sqrt{2}} \int_{0}^{\tau}\|\tilde{\mathbf{e}}(\sigma) \psi\| \mathrm{d} \sigma .
$$

It follows, in particular, that

$$
\|\tilde{\boldsymbol{\pi}}(\tau) \psi\| \leq \sqrt{2}\left\|h(0)^{1 / 2} \psi\right\|+\int_{0}^{\tau}\|\mathbf{e}(\sigma, .)\|_{\infty} \mathrm{d} \sigma\|\psi\| .
$$

Theorem 5. Let the electromagnetic fields and potentials satisfy the assumptions of Theorem 3 in the form given in Proposition 4 (iii). If the field $\mathbf{e}$ satisfies

$$
\int_{-\pi / 2}^{\pi / 2}\left(\frac{\pi}{2}-|\tau|\right)\|\mathbf{e}(\tau, .)\|_{\infty} \mathrm{d} \tau<\infty
$$

then there exist the asymptotic position operators $\tilde{\mathbf{x}}_{ \pm}$defined by

$$
\exp \left[i \mathbf{q} \cdot \tilde{\mathbf{x}}_{ \pm}\right]=\underset{\tau \rightarrow \pm \pi / 2}{\mathrm{~s}-\lim _{\tau \rightarrow \pi}} \exp [i \mathbf{q} \cdot \tilde{\mathbf{x}}(\tau)]
$$

for all numerical vectors $\mathbf{q}$. The limit then holds for any Schwartz function.

Proof. Using equation (23) we obtain

$$
\left\|\partial_{\tau} \exp [i \mathbf{q} \cdot \tilde{\mathbf{x}}(\tau)] \psi\right\|=\left\|\left[\mathbf{q} \cdot \tilde{\boldsymbol{\pi}}(\tau)+\frac{1}{2} \mathbf{q}^{2}\right] \psi\right\| \leq|\mathbf{q}|\|\tilde{\boldsymbol{\pi}}(\tau) \psi\|+\frac{1}{2}|\mathbf{q}|^{2}\|\psi\| .
$$

Noting that the integration by parts gives

$$
\int_{0}^{\pi / 2} \int_{0}^{\tau}\|\mathbf{e}(\sigma, .)\|_{\infty} \mathrm{d} \sigma \mathrm{d} \tau \leq \int_{0}^{\pi / 2}\left(\frac{\pi}{2}-\tau\right)\|\mathbf{e}(\tau, .)\|_{\infty} \mathrm{d} \tau
$$

we observe that the RHS of (24) and (26) are integrable over $[0, \pi / 2]$. Therefore, the $\operatorname{limit} \lim _{\tau \rightarrow \pi / 2} \exp [i \mathbf{q} \cdot \tilde{\mathbf{x}}(\tau)] \psi$ exists for each $\psi \in \mathcal{D}\left(h_{0}\right)$, hence for 
all $\psi$. The existence of a self-adjoint operator $\tilde{\mathbf{x}}_{+}$satisfying the limiting relation (25) follows. ${ }^{7}$ The case of $\tilde{\mathbf{x}}_{-}$is similar.

We now turn to the existence of wave operators.

Theorem 6. Let the electromagnetic fields and potentials satisfy again the assumptions given in Proposition 4 (iii). Suppose that

$$
\int_{-\pi / 2}^{\pi / 2}\left(\frac{\pi}{2}-|\tau|\right)^{2}\left\|\mathbf{e}(\tau, .)^{2}\right\|_{\infty} \mathrm{d} \tau<\infty
$$

and for each closed set $K \subset \mathbb{R}^{3} \backslash\left\{\mathbf{x}: \mathbf{x}^{2}=1\right\}$, with notation $\|\cdot\|_{K, \infty} \equiv$ $\|\cdot\|_{L^{\infty}(K)}$, there is

$$
\int_{-\pi / 2}^{\pi / 2}\left(\frac{\pi}{2}-|\tau|\right)\|\boldsymbol{\partial} \cdot \mathbf{e}(\tau, .)\|_{K, \infty} \mathrm{d} \tau<\infty .
$$

Then there exist isometric wave operators

$$
u(0, \pm \pi / 2) \equiv \underset{\tau \rightarrow \pm \pi / 2}{\mathrm{~s}-\lim _{\tau \rightarrow \pi}} u(0, \tau) \equiv \omega_{ \pm},
$$

such that for each bounded, continuous function $f(\mathbf{x})$ with support not intersecting $\mathbf{x}^{2}=1$ there is

$$
f\left(\tilde{\mathbf{x}}_{ \pm}\right)=\omega_{ \pm} f(\mathbf{x}) \omega_{ \pm}^{*}
$$

It follows that

$$
\omega_{ \pm} \omega_{ \pm}^{*}=E_{ \pm} \equiv \mathbb{1}-\mathbb{1}_{\{1\}}\left(\tilde{\mathbf{x}}_{ \pm}^{2}\right)
$$

and

$$
\underset{\tau \rightarrow \pm \pi / 2}{\mathrm{~S}-\lim _{\tau}} u(\tau, 0) E_{ \pm}=\omega_{ \pm}^{*}
$$

Proof. Let $\psi$ be any smooth function with compact support $K$ outside $\mathbf{x}^{2}=1$. If we show that $\|h(\tau) \psi\|$ is integrable over $[-\pi / 2, \pi / 2]$, then by Eq. (7) $u(0, \tau) \psi$ converges for $\tau \rightarrow \pm \pi / 2$. As the assumed class of functions is dense in $\mathcal{H}$, the existence of $\omega_{ \pm}$will be achieved. Now,

$$
\begin{gathered}
\|h(\tau) \psi\| \leq\left\|h_{0} \psi\right\|+\|\mathbf{a}(\tau) \cdot \boldsymbol{\partial} \psi\|+\frac{1}{2}\|(\boldsymbol{\partial} \cdot \mathbf{a})(\tau) \psi\|+\frac{1}{2}\left\|\mathbf{a}(\tau)^{2} \psi\right\| \\
\leq\left\|h_{0} \psi\right\|+\|\mathbf{a}(\tau, .)\|_{\infty}\|\boldsymbol{\partial} \psi\| \\
\quad+\frac{1}{2}\left(\|\mathbf{a}(\tau, .)\|_{\infty}^{2}+\|\boldsymbol{\partial} \cdot \mathbf{a}(\tau, .)\|_{K, \infty}\right)\|\psi\| .
\end{gathered}
$$

Using the Schwarz inequality and the gauge (13), we have

$$
\frac{2}{\pi}\left[\int_{0}^{\pi / 2}\|\mathbf{a}(\tau, .)\|_{\infty} \mathrm{d} \tau\right]^{2} \leq \int_{0}^{\pi / 2}\|\mathbf{a}(\tau, .)\|_{\infty}^{2} \mathrm{~d} \tau \leq \pi\|\mathbf{a}(0, .)\|_{\infty}^{2}+2 \int_{0}^{\pi / 2} n(\tau)^{2} \mathrm{~d} \tau,
$$

\footnotetext{
${ }^{7}$ This is a simple consequence, which is left to the reader as Problem VIII.23 in [9].
} 
where $n(\tau)=\int_{0}^{\tau}\|\mathbf{e}(\sigma, .)\|_{\infty} \mathrm{d} \sigma$. Integrating by parts and then using the Schwarz inequality, we obtain

$$
\begin{aligned}
& \int_{0}^{\pi / 2} n(\tau)^{2} \mathrm{~d} \tau \leq 2 \int_{0}^{\pi / 2}\left(\frac{\pi}{2}-\tau\right) n(\tau) \dot{n}(\tau) \mathrm{d} \tau \\
& \quad \leq 2 \sqrt{\int_{0}^{\pi / 2} n(\tau)^{2} \mathrm{~d} \tau} \sqrt{\int_{0}^{\pi / 2}\left(\frac{\pi}{2}-\tau\right)^{2} \dot{n}(\tau)^{2} \mathrm{~d} \tau} \\
& \quad \leq 4 \int_{0}^{\pi / 2}\left(\frac{\pi}{2}-\tau\right)^{2}\|\mathbf{e}(\tau, .)\|_{\infty}^{2} \mathrm{~d} \tau,
\end{aligned}
$$

which shows that the second and third terms on the RHS of (33) are integrable over $[0, \pi / 2]$. Finally, integrating by parts as in (27) we find

$\int_{0}^{\pi / 2}\|\boldsymbol{\partial} \cdot \mathbf{a}(\tau, .)\|_{K, \infty} \mathrm{d} \tau \leq \frac{\pi}{2}\|\boldsymbol{\partial} \cdot \mathbf{a}(0, .)\|_{K, \infty}+\int_{0}^{\pi / 2}\left(\frac{\pi}{2}-\tau\right)\|\boldsymbol{\partial} \cdot \mathbf{e}(\tau, .)\|_{K, \infty} \mathrm{d} \tau$, which completes the proof of the existence of $\omega_{+}(29)$. The case of $\omega_{-}$is analogous.

The proof of the remaining claims is based on the following property, to be proved below: for each smooth function $f(\mathbf{x})$ with compact support $K$ not intersecting $\mathbf{x}^{2}=1$, there is

$$
\underset{\tau \rightarrow \pm \pi / 2}{\mathrm{~S}-\lim _{\tau \rightarrow \pi}} f(\mathbf{x}) u(\tau, 0)=f(\mathbf{x}) \omega_{ \pm}^{*} .
$$

Once this is proved, we have

$$
f\left(\tilde{\mathbf{x}}_{ \pm}\right)=\underset{\tau \rightarrow \pm \pi / 2}{\mathrm{~s}-\lim _{\tau}} u(0, \tau) f(\mathbf{x}) u(\tau, 0)=\omega_{ \pm} f(\mathbf{x}) \omega_{ \pm}^{*}
$$

where the first equality is the result of Theorem 5. Each bounded continuous function with support outside $\mathbf{x}^{2}=1$, as well as the characteristic function of the set $\mathbb{R}^{3} \backslash\left\{\mathbf{x} \mid \mathbf{x}^{2}=1\right\}$, is a point-wise limit of the above functions, so (30) and (31) follow (for the latter note that $\mathbb{1}_{\{1\}}\left(\mathbf{x}^{2}\right)=0$ ). Finally, there is

$$
\left\|u(\tau, 0) E_{ \pm} \psi-\omega_{ \pm}^{*} \psi\right\|^{2}=2\left\|E_{ \pm} \psi\right\|^{2}-2 \operatorname{Re}\left(E_{ \pm} \psi, u(0, \tau) \omega_{ \pm}^{*} \psi\right) \rightarrow 0
$$

for $\tau \rightarrow \pm \pi / 2$, so (32) follows.

Turning to the proof of the remaining property (34) we use (6) to obtain for $\psi \in \mathcal{D}\left(h_{0}\right)$ :

$$
u_{0}(0, \tau) f(\mathbf{x}) u(\tau, 0) \psi=f(\mathbf{x}) \psi-i \int_{0}^{\tau} u_{0}(0, \sigma)\left[f h(\sigma)-h_{0} f\right] u(\sigma, 0) \psi \mathrm{d} \sigma,
$$

where $u_{0}$ and $h_{0}$ are unperturbed operators, and without restricting generality we assume $\|f\|_{\infty} \leq 1$. Now,

$$
f h-h_{0} f=(i \partial f-f \mathbf{a}) \cdot \boldsymbol{\pi}+\frac{1}{2} f\left(i \boldsymbol{\partial} \cdot \mathbf{a}-\mathbf{a}^{2}\right)+i(\boldsymbol{\partial f} \cdot \mathbf{a})+\frac{1}{2}(\Delta f) .
$$

The norm of the integrand in (35) may be thus bounded as

$$
\begin{aligned}
& \left\|\left[f h(\sigma)-h_{0} f\right] u(\sigma, 0) \psi\right\| \leq\left(\|\boldsymbol{\partial} f\|_{\infty}+\|\mathbf{a}(\sigma, .)\|_{\infty}\right)\|\tilde{\boldsymbol{\pi}}(\sigma) \psi\| \\
& \quad+\frac{1}{2}\left[\|\boldsymbol{\partial} \cdot \mathbf{a}(\sigma, .)\|_{K, \infty}+\|\mathbf{a}(\sigma, .)\|_{\infty}^{2}+2\|\boldsymbol{\partial} f\|_{\infty}\|\mathbf{a}(\sigma, .)\|_{\infty}+\|\Delta f\|_{\infty}\right]\|\psi\| .
\end{aligned}
$$


The integrability on $[0, \pi / 2]$ of the terms in the second line follows from the proof of the existence of $\omega_{+}$, and of the first term in the first line-from the proof of Theorem 5. Finally, the proof of integrability of $\|\mathbf{a}(\sigma, .)\|_{\infty}\|\tilde{\boldsymbol{\pi}}(\sigma) \psi\|$ is very similar to the case of $\|\mathbf{a}(\sigma, .)\|_{\infty}^{2}$ (see relations (13) and (24)). This shows that the LHS of (35) has a limit for $\tau \rightarrow \pi / 2$. But $u_{0}(0, \tau)$ has a unitary strong limit operator $u_{0}(0, \pi / 2)$, so $f(\mathbf{x}) u(\tau, 0) \psi$ converges strongly to some vector $\chi$. It follows that for each vector $\varphi$ there is

$$
\begin{aligned}
& (\varphi, \chi)=\lim _{\tau \rightarrow \pi / 2}(\varphi, f(\mathbf{x}) u(\tau, 0) \psi)=\lim _{\tau \rightarrow \pi / 2}\left(u(0, \tau) f(\mathbf{x})^{*} \varphi, \psi\right)=\left(\omega_{+} f(\mathbf{x})^{*} \varphi, \psi\right), \\
& \text { so } \chi=f(\mathbf{x}) \omega_{+}^{*} \psi \text { and the property (34) follows. }
\end{aligned}
$$

It should be clear from the proof of the above theorem that the only potential obstacle to the asymptotic completeness is the behavior of $\boldsymbol{\partial} \cdot \mathbf{e}(\tau, \mathbf{x})$ in the neighborhood of $\mathbf{x}^{2}=1$. If the norm $\|\boldsymbol{\partial} \cdot \mathbf{e}(\tau, .)\|_{K, \infty}$ may be replaced by $\|\boldsymbol{\partial} \cdot \mathbf{e}(\tau, .)\|_{\infty}$, then in the proof of convergence of (35) the function $f$ may be replaced by 1 , and one obtains the following.

Theorem 7. If the assumptions of Theorem 6 hold with the condition (28) replaced by

$$
\int_{-\pi / 2}^{\pi / 2}\left(\frac{\pi}{2}-|\tau|\right)\|\boldsymbol{\partial} \cdot \mathbf{e}(\tau, .)\|_{\infty} \mathrm{d} \tau<\infty,
$$

then the wave operators $\omega_{ \pm}$are unitary.

\section{Back to Spacetime and Conclusions}

Let us remind the reader, that the class of scattering electromagnetic fields $F(x)$ was announced in the abstract, and then discussed in Appendix B. The bounds they satisfy were adapted to the field e in Sect. 5, where we also anticipated that the resulting estimates satisfy the assumptions of Theorems 5 and 6 . We now formulate the results of these theorems in natural spacetime terms, with the use of relation (10) providing the link between the oscillator picture evolution operator $u(\tau, \sigma)$ and the Schrödinger operator $U(t, s)$.

The asymptotic variables $\tilde{\mathbf{x}}_{ \pm}$provided by Theorem 5 may be obtained by

$$
\exp \left[i \mathbf{q} \cdot \tilde{\mathbf{x}}_{ \pm}\right]=\underset{t \rightarrow \pm \infty}{\mathrm{s}-\lim _{i \rightarrow \infty}} U(0, t) \exp \left[i \mathbf{q} \cdot \frac{\mathbf{x}}{\langle t\rangle}\right] U(t, 0),
$$

where we used relation (12). Now they have a natural interpretation of asymptotic velocity operators. The wave operators $\omega_{ \pm}$of Theorem 6 are now

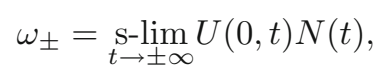

and their conjugates are given by

$$
\omega_{ \pm}^{*}=\underset{t \rightarrow \pm \infty}{\operatorname{s}-\lim _{t}} N(t)^{*} U(t, 0) E_{ \pm} .
$$

It is visible that with our choice of gauge the definition of wave operators in the present formalism does not need further corrections (e.g., of the Dollard 
type) to compensate the long-range character of the interaction. The joint spectrum of $\tilde{\mathbf{x}}_{+}$(and similarly $\tilde{\mathbf{x}}_{-}$) covers the whole space $\mathbb{R}^{3}$ and outside $\tilde{\mathbf{x}}_{+}^{2}=1\left(\tilde{\mathbf{x}}_{-}^{2}=1\right)$ is absolutely continuous. Whether this continuity extends to $\mathbb{R}^{3}$, in which case $E_{ \pm}=\mathbb{1}$ and the asymptotic completeness holds, is an open problem. The difficulty lies in the null-asymptotic behavior of electromagnetic potentials (and fields), which is slower than assumed in usual analyses of timedecaying potentials. The problem may be due to the usual inconsistency in systems of the considered type: the Lorentz symmetry of the electromagnetic field vs Galilean symmetry of the Schrödinger equation.

The existence of the wave operators in the given form, despite the problems with asymptotic completeness, is a further argument for our main point: appropriate choice of gauge eliminates at least some of the infrared problems. The gauge condition found suitable in the present context is characterized by Eq. (14). This property closely parallels the gauge condition obtained in the analysis of the asymptotic behavior of the Dirac field inside the lightcone [4]. In our opinion, this may have interesting implications for quantum electrodynamics as well, although in that context the non-locality of the gauge will be a problem (cf. (13)).

\section{Acknowledgements}

I am grateful to Jan Dereziński for important literature hints and to Paweł Duch for an interesting discussion.

Open Access. This article is distributed under the terms of the Creative Commons Attribution 4.0 International License (http://creativecommons.org/licenses/ by/4.0/), which permits unrestricted use, distribution, and reproduction in any medium, provided you give appropriate credit to the original author(s) and the source, provide a link to the Creative Commons license, and indicate if changes were made.

Publisher's Note Springer Nature remains neutral with regard to jurisdictional claims in published maps and institutional affiliations.

\section{Appendix A: Domain Relations}

Lemma 8. Let $\mathbf{a}$, $\boldsymbol{\partial \mathbf { a }} \in L^{\infty}\left(\mathbb{R}^{3}\right)$, so that the operator $\boldsymbol{\pi}^{2}+\mathbf{x}^{2}$ is essentially self-adjoint on $C_{0}^{\infty}\left(\mathbb{R}^{3}\right)$. Then the following inequalities are satisfied

$$
\begin{aligned}
& \left\|\boldsymbol{\pi}^{2} \varphi\right\|^{2}+\left\|\mathbf{x}^{2} \varphi\right\|^{2} \leq\left\|\left(\boldsymbol{\pi}^{2}+\mathbf{x}^{2}\right) \varphi\right\|^{2}+6\|\varphi\|^{2} \\
& \|(\mathbf{x} \cdot \boldsymbol{\pi}+\boldsymbol{\pi} \cdot \mathbf{x}) \varphi\|^{2} \leq 2\left(\left\|\boldsymbol{\pi}^{2} \varphi\right\|^{2}+\left\|\mathbf{x}^{2} \varphi\right\|^{2}\right)+3\|\varphi\|^{2} .
\end{aligned}
$$

Therefore,

$$
\mathcal{D}\left(\boldsymbol{\pi}^{2}\right) \cap \mathcal{D}\left(\mathbf{x}^{2}\right)=\mathcal{D}\left(\boldsymbol{\pi}^{2}+\mathbf{x}^{2}\right) \subseteq \mathcal{D}(\mathbf{x} \cdot \boldsymbol{\pi}+\boldsymbol{\pi} \cdot \mathbf{x}) .
$$

Proof. For $\varphi \in C_{0}^{\infty}\left(\mathbb{R}^{3}\right)$ the following identity is easily obtained:

$$
\bar{\varphi} \boldsymbol{\pi}^{2} \varphi+\varphi \overline{\pi^{2} \varphi}=-\Delta|\varphi|^{2}+2|\boldsymbol{\pi} \varphi|^{2} .
$$


Multiplying this by $\mathbf{x}^{2}$ and integrating, one obtains (integrate by parts the first term on the RHS)

$$
\operatorname{Re}\left(\boldsymbol{\pi}^{2} \varphi, \mathbf{x}^{2} \varphi\right)=-3\|\varphi\|^{2}+\||\mathbf{x}| \boldsymbol{\pi} \varphi\|^{2} \geq-3\|\varphi\|^{2},
$$

so (36) for $\varphi \in C_{0}^{\infty}\left(\mathbb{R}^{3}\right)$ easily follows. For (37) note that $|\mathbf{x} \cdot \boldsymbol{\pi} \varphi|^{2} \leq \mathbf{x}^{2}|\boldsymbol{\pi} \varphi|^{2}$, so using again (38) we have

$$
\begin{aligned}
& \|\mathbf{x} \cdot \boldsymbol{\pi} \varphi\|^{2} \leq \frac{1}{2} \int \mathbf{x}^{2} \Delta|\varphi|^{2}+\operatorname{Re}\left(\boldsymbol{\pi}^{2} \varphi, \mathbf{x}^{2} \varphi\right) \\
& \quad \leq 3\|\varphi\|^{2}+\left\|\pi^{2} \varphi\right\|\left\|\mathbf{x}^{2} \varphi\right\| \leq \frac{1}{2}\left(\left\|\pi^{2} \varphi\right\|^{2}+\left\|\mathbf{x}^{2} \varphi\right\|^{2}\right)+3\|\varphi\|^{2} .
\end{aligned}
$$

Moreover, splitting $\mathbf{x} \cdot \boldsymbol{\pi}=\frac{1}{2}(\mathbf{x} \cdot \boldsymbol{\pi}+\boldsymbol{\pi} \cdot \mathbf{x})+\frac{3}{2} i$ one has

$$
\|\mathbf{x} \cdot \boldsymbol{\pi} \varphi\|^{2}=\frac{1}{4}\|(\mathbf{x} \cdot \boldsymbol{\pi}+\boldsymbol{\pi} \cdot \mathbf{x}) \varphi\|^{2}+\frac{9}{4}\|\varphi\|^{2} .
$$

Substituting this on the LHS of the last inequality one obtains (37) for $\varphi \in C_{0}^{\infty}\left(\mathbb{R}^{3}\right)$.

If $\varphi \in \mathcal{D}\left(\boldsymbol{\pi}^{2}+\mathbf{x}^{2}\right)$, then it is approximated in the graph norm of this operator by a sequence $\varphi_{n} \in C_{0}^{\infty}\left(\mathbb{R}^{3}\right)$. The inequalities (36) and (37) show that then this is also a Cauchy sequence in the graph norms of $\boldsymbol{\pi}^{2}, \mathbf{x}^{2}$ and $\mathbf{x} \cdot \boldsymbol{\pi}+\boldsymbol{\pi} \cdot \mathbf{x}$. These operators are closed, so $\varphi$ is in their domains, and relations (36) and (37) are satisfied on $\mathcal{D}\left(\boldsymbol{\pi}^{2}+\mathbf{x}^{2}\right)$. The inclusion $\mathcal{D}\left(\boldsymbol{\pi}^{2}\right) \cap \mathcal{D}\left(\mathbf{x}^{2}\right) \subseteq \mathcal{D}\left(\boldsymbol{\pi}^{2}+\mathbf{x}^{2}\right)$ is obvious.

\section{Appendix B: Asymptotic Behavior of Electromagnetic Fields}

We discuss here the decay properties of electromagnetic fields in the Minkowski spacetime language. For more extensive discussion and explanation of details we refer the reader to $[6]$.

Fields $\mathbf{E}$ and $\mathbf{B}$ are the 3-vector parts of the Minkowski tensor $F_{a b}(x)$ given by

$$
E^{i}(t, \mathbf{x})=F^{i 0}(x), \quad B^{i}(t, \mathbf{x})=-\sum_{j k} \varepsilon^{i j k} F^{j k}(x) ;
$$

here and in the rest of these remarks: $x=(t, \mathbf{x}) ; a, b$ are Minkowski indices and $i, j, k$ are 3 -space indices. Also, we note that the field $\mathbf{C}$ defined by (15) is the 3 -vector space part of the field

$$
C^{a}(x)=F^{a}{ }_{b}(x) x^{b} .
$$

Electromagnetic fields present in scattering situations of the field theory have the form

$$
F=F^{\mathrm{ret}}+F^{\mathrm{in}}=F^{\mathrm{adv}}+F^{\mathrm{out}},
$$

where $F^{\mathrm{ret}} / F^{\mathrm{adv}}$ is the retarded/advanced field of an electromagnetic current:

$$
F_{a b}^{\mathrm{ret} / \mathrm{adv}}(x)=4 \pi \int D_{\mathrm{ret} / \mathrm{adv}}(x-y)\left[\partial_{a} J_{b}(y)-\partial_{b} J_{a}(y)\right] \mathrm{d} y
$$

and $F^{\text {in }} / F^{\text {out }}$ is a free incoming/outgoing field. In remote past and future, the current $J$ is assumed to tend to asymptotic currents, homogeneous of 
degree -3 , with support inside the lightcone. Similarly, the free fields are produced as radiation fields of currents with the asymptotic behavior of the same type. We discuss the asymptotic behavior of fields in the half-space $t \geq 0$ with the use of representation $F=F^{\text {adv }}+F^{\text {out }}$; the case $t \leq 0$ with $F=$ $F^{\text {ret }}+F^{\text {in }}$ is analogous.

For $t \geq 0$ and $t+|\mathbf{x}|$ tending to infinity, the dominant contribution to the advanced field comes from the asymptotic outgoing current. Therefore, in this region this field is homogeneous of degree -2 , hence the field $\mathbf{C}^{\mathrm{adv}}$ is homogeneous of degree -1 , and the field $\boldsymbol{\partial} \cdot \mathbf{C}^{\text {adv }}$ is homogeneous of degree -2 . Thus for the advanced part the bounds (17) and (18) are satisfied. In fact, the -2 homogeneity of $\boldsymbol{\partial} \cdot \mathbf{C}^{\text {adv }}$ implies that for the corresponding part $\mathbf{e}^{\text {adv }}$ of $\mathbf{e}$ there is $\left|\boldsymbol{\partial} \cdot \mathbf{e}^{\operatorname{adv}}(\tau, \mathbf{x})\right| \leq$ const $/ \cos \tau$, which is a bound of the type (20) sufficient for the validity of Theorem $7 .^{8}$

For the free outgoing field and its Lorenz gauge potential, we use the integral representations valid for solutions of the wave equation (see, e.g., [6]):

$$
\begin{aligned}
& F_{a b}^{\text {out }}(x)=-\frac{1}{2 \pi} \int\left[l_{a} \ddot{V}_{b}(x \cdot l, l)-l_{b} \ddot{V}_{a}(x \cdot l, l)\right] \mathrm{d}^{2} l, \\
& A_{a}^{\text {out }}(x)=-\frac{1}{2 \pi} \int \dot{V}_{a}(x \cdot l, l) \mathrm{d}^{2} l .
\end{aligned}
$$

Here $l$ represents a vector on the future lightcone and $d^{2} l$ is the Lorentzinvariant measure on the set of future null directions, applicable to functions of $l$ homogeneous of degree -2 . The function $V$ describes the future null asymptote of the potential and the field:

$$
\lim _{R \rightarrow \infty} R A^{\text {out }}(x+R l)=V(x \cdot l, l), \quad \lim _{R \rightarrow \infty} R F^{\text {out }}(x+R l)=2 l \wedge \dot{V}(x \cdot l, l),
$$

and $\dot{V}(s, l)=\partial V(s, l) / \partial s$. Moreover, $V(s, l)$ is homogeneous of degree -1 , $l \cdot V(s, l)=0, V(s, l) \rightarrow 0$ for $s \rightarrow \infty$, and $\dot{V}(s, l)$ (together with its low orders derivatives in the cone variable) is bounded by const $(1+|s|)^{-1-\varepsilon}$ for some $\varepsilon>0$. Using this bound, it is easy to show that

$$
\begin{aligned}
\left|A^{\text {out }}(x)\right| & \leq \text { const } \int \frac{\mathrm{d} \Omega(\mathbf{l})}{\left(1+\left|x^{0}-\mathbf{x} \cdot \mathbf{l}\right|\right)^{1+\varepsilon}} \\
& \leq \frac{\text { const }}{1+\left|x^{0}\right|+|\mathbf{x}|}\left[\frac{\theta\left(x^{2}\right)}{\left(1+\left|x^{0}\right|-|\mathbf{x}|\right)^{\varepsilon}}+\theta\left(-x^{2}\right)\right]
\end{aligned}
$$

where $\mathbf{l}$ represents unit 3 -vectors and $d \Omega(\mathbf{l})=\sin \vartheta d \vartheta d \varphi$ is the solid angle measure, with $\vartheta$ measured with respect to $\mathbf{x}$. In the region $x^{2} \geq 0$ the field $F^{\text {out }}$ is estimated similarly as above (with the bound of $\ddot{V}(s, l)$ by const $(1+|s|)^{-2-\varepsilon}$ following from earlier assumptions), but in the region $x^{2}<0$ one first has to

\footnotetext{
${ }^{8}$ The above reasoning is sufficient as it stands if the asymptotic currents do not have oscillating contributions. However, a closer analysis of the cases such as the classical Dirac field current, which does have oscillating asymptotic terms, confirms the above bounds also in this case. We do not enter here into more detailed discussion of this point and refer the reader to $[4,6]$ for a more explicit discussion of the setting.
} 
integrate in (40) once by parts with respect to $\vartheta$ angle variable. In this way one finds

$$
\left|F^{\text {out }}(x)\right| \leq \frac{\text { const }}{1+\left|x^{0}\right|+|\mathbf{x}|}\left[\frac{1}{\left(1+|| x^{0}|-| \mathbf{x}||\right)^{1+\varepsilon}}+\frac{\theta\left(-x^{2}\right)}{1+\left|x^{0}\right|+|\mathbf{x}|}\right] .
$$

The estimate of $\mathbf{E}^{\text {out }}$ in (17) is obviously satisfied. We turn to the field $\mathbf{C}^{\text {out }}$. The relation (39) shows that this is again a solution of the wave equation with the integral representation

$$
C_{a}^{\text {out }}(x)=-\frac{1}{2 \pi} \int \dot{W}_{a}(x \cdot l, l) \mathrm{d}^{2} l .
$$

Denote $L_{a b}=l_{a} \partial / \partial l^{b}-l_{b} \partial / \partial l^{a}$-the intrinsic differential operator on the lightcone. With the use of identity $\int L_{a b} F(l) d^{2} l=0$ valid for each $C^{1}$-function $F$, homogeneous of degree -2 , one shows that

$$
W^{a}(s, l)=V^{a}(s, l)-s \dot{V}^{a}(s, l)-L^{a b} V_{b}(s, l) .
$$

As $l \cdot W(s, l)=0$, one has $\partial \cdot C(x)=0$, so

$$
\partial \cdot \mathbf{C}^{\text {out }}(x)=\frac{1}{2 \pi} \int l_{0} \ddot{W}^{0}(x \cdot l, l) \mathrm{d}^{2} l .
$$

Now, it is easy to see that the function $\dot{W}(s, l)$ has the same decay properties in $|s|$ as function $\dot{V}(s, l)$, so $\mathbf{C}^{\text {out }}(x)$ and $\boldsymbol{\partial} \cdot \mathbf{C}^{\text {out }}(x)$ are similarly estimated as $A^{\text {out }}(x)$ and $F^{\text {out }}(x)$, respectively. This is sufficient to satisfy the bounds (17) and (18).

\section{Appendix C: Differentiation of Operators $\tilde{\boldsymbol{k}}$}

For a symmetric operator $\tilde{k}$ defined by (21) suppose that $\mathcal{D}\left(h_{0}\right)$ is contained in the domain of $\dot{k}$ and of the weak commutator $[k(\tau), h(\tau)]_{\mathrm{w}}$ defined for $\varphi, \psi \in$ $\mathcal{D}\left(h_{0}\right)$ by:

$$
\left(\varphi,[k(\tau), h(\tau)]_{\mathrm{w}} \psi\right)=(k(\tau) \varphi, h(\tau) \psi)-(h(\tau) \varphi, k(\tau) \psi) .
$$

In this case, we have $\partial_{\tau}(\varphi, \tilde{k} \psi)=\left(\varphi,\left(\tilde{\dot{k}}-i \widetilde{[k, h]_{\mathrm{w}}}\right) \psi\right)$, and upon integrating, we can omit the product with $\varphi$ to obtain

$$
[\tilde{k}(\tau)-k(0)] \psi=\int_{0}^{\tau}\left(\tilde{\dot{k}}-i \widetilde{[k, h]_{\mathrm{w}}}\right)(\rho) \psi \mathrm{d} \rho,
$$

from which the differential form follows

$$
\dot{\tilde{k}} \psi=\left(\tilde{\dot{k}}-i \widetilde{[k, h]_{\mathrm{w}}}\right) \psi
$$

For our basic observables, it is easy to show, that the weak commutators are equal to those calculated naively, so in particular

$$
[\mathbf{x}, h]_{\mathrm{w}}=i \boldsymbol{\pi} .
$$

Also, rather obviously, $[h, h]_{\mathrm{w}}=0$, so $\dot{\tilde{h}}=\tilde{\dot{h}}$. 


\section{References}

[1] Duch, P.: Weak adiabatic limit in quantum field theories with massless particles. Ann. H. Poincaré 19, 875-935 (2018)

[2] Dereziński, J., Gérard, C.: Scattering Theory of Classical and Quantum NParticle Systems. Springer, Berlin (1997)

[3] Dereziński, J., Gérard, C.: Long-range scattering in the position representation. J. Math. Phys. 38, 3925-3942 (1997)

[4] Herdegen, A.: Long-range effects in asymptotic fields and angular momentum of classical field electrodynamics. J. Math. Phys. 36, 4044-4086 (1995)

[5] Herdegen, A.: Semidirect product of CCR and CAR algebras and asymptotic states in quantum electrodynamics. J. Math. Phys. 39, 1788-1817 (1998)

[6] Herdegen, A.: Asymptotic structure of electrodynamics revisited. Lett. Math. Phys. 107, 1439-1470 (2017)

[7] Leinfelder, H., Simader, C.: Schrödinger operators with singular magnetic vector potentials. Math. Zeitschr. 176, 1-19 (1981)

[8] Niederer, U.: The maximal kinematical invariance group of the harmonic oscillator. Helv. Phys. Acta 46, 191-200 (1970)

[9] Reed, M., Simon, B.: Methods of Modern Mathematical Physics, vol. I. Academic Press, San Diego (1975)

[10] Reed, M., Simon, B.: Methods of Modern Mathematical Physics, vol. II. Academic Press, San Diego (1979)

[11] Yafaev, D.R.: Mathematical Scattering Theory. Analytic Theory. American Mathematical Society, Providence (2010)

[12] Yajima, K.: Schrödinger equations with time-dependent unbounded singular potentials. Rev. Math. Phys. 23, 823-838 (2011)

Andrzej Herdegen

Institute of Physics

Jagiellonian University

ul. S. Łojasiewicza 11

30-348 Kraków

Poland

e-mail: herdegen@th.if.uj.edu.pl

Communicated by Jan Derezinski.

Received: May 31, 2019.

Accepted: September 19, 2019. 